\title{
ABORDAGEM CIRÚRGICA DE HEMANGIOMA CAVERNOSO EM LÍNGUA: RELATO DE CASO
}

\author{
Raissa Faria ANDRADE ${ }^{1}$ \\ Flávia de Oliveira SILVA ${ }^{1}$ \\ Rafael de Aguiar VILELA JR ${ }^{2}$ \\ Márcio Américo DIAS ${ }^{3}$ \\ Lucinei Roberto OLIVEIRA ${ }^{4}$
}

\begin{abstract}
${ }^{1}$ Acadêmicas de Odontologia/ INAPÓS - Pouso Alegre-MG (raissafandrade@ gmail.com e flaviiasilva@ hotmail.com)
${ }^{2}$ Cirurgião-Dentista. Mestrando em Clínica Odontológica (UninCor). Docente do curso de Odontologia/ INAPÓS Pouso Alegre-MG (rafaelvilelajr@yahoo.com.br)

${ }^{3}$ Cirurgião-Dentista. Mestre em Clínica Odontológica (UninCor). Docente do curso de Odontologia/ INAPÓS - Pouso Alegre-MG (marcdias6@gmail.com)

${ }^{4}$ Cirurgião-Dentista. Mestre em Estomatopatologia (FOP/UNICAMP). Doutor e Pós-Doutor em Patologia (FMRP/USP). Docente dos cursos de Odontologia do INAPÓS - Pouso Alegre-MG e da UninCor - Três CoraçõesMG(lucinei@yahoo.com)
\end{abstract}

Recebido em: 24/05/2014 - Aprovado em: 30/06/2014 - Disponibilizado em: 30/07/2014

\section{RESUMO:}

O hemangioma cavernoso (HC) é considerado uma neoplasia benigna vascular, incluindo como característica a proliferação de vasos sanguíneos, mas constantemente também aparece como uma anomalia de desenvolvimento, sendo chamado de hamartoma. São lesões que podem ocorrer em qualquer faixa etária, após o nascimento ou nos decorridos primeiros anos de vida, principalmente em pacientes do gênero masculino. Tais lesões são persistentes e às vezes mal definidas, podendo também acometer a pele, as membranas mucosas e osso. Ocorrem predominantemente em região de cabeça e pescoço, sendo que na cavidade oral acometem frequentemente os lábios, a língua e a mucosa jugal. O HC é caracterizado por apresentar-se como uma mácula ou nódulo, cuja coloração varia de vermelho intenso ao roxo, de acordo com sua localização e profundidade no tecido, além do grau de congestão do mesmo. Seu tamanho pode ser variável, dependendo da idade do paciente e da localização. A erradicação do HC é geralmente difícil, pois as recidivas são comuns, permanecendo o seu tratamento ainda em controverso entre autores. Junto com uma revisão da literatura atual sobre o tema, o presente artigo teve como objetivo relatar um caso clínico de um hemangioma em língua, com remoção por enucleação e ausência de recidiva após seis meses.

Palavras-chave: Hemangioma. Neoplasia Vascular. Hamartoma. Anomalia de desenvolvimento. Diascopia.

\section{ABSTRACT:}

The cavernous hemangioma $(\mathrm{CH})$ is considered a benign vascular neoplasm, characterized by blood vessel proliferation, but constantly also appears as a development anomaly, called hamartoma. The lesions can occur at any age, but the diagnosis occurs predominantly after birth or in early life, especially in male patients. Such lesions are persistent and sometimes indefined, and can affect the skin, mucous membranes and bone. The $\mathrm{CH}$ occurs predominantly in the head and neck areas, and in the oral cavity often affect lips, tongue and the buccal mucosa. The $\mathrm{CH}$ is characterized by presenting itself as lump, whose color can range from deep red to purple, according to the location and depth in the tissue, besides the degree of tissular congestion. Its size varies depending on location and age of the patient. The $\mathrm{CH}$ elimination is sometimes difficult, and recurrences are common. The $\mathrm{CH}$ treatment still remains controversial among authors. Along with a review of the current literature on the topic, this paper aims report a case of a hemangioma in tongue removed through surgical enucleation, that still have showed none recurrences after six months. Keywords: Hemangioma. Vascular neoplasia. Hamartoma. Developmental anomaly. Diascopy. 


\section{INTRODUÇÃO}

Atualmente, o hemangioma é referido pela OMS - Organização Mundial de Saúde como uma neoplasia vascular benigna em fase de crescimento rápido com proliferação anormal de vasos sanguíneos e células endoteliais, podendo ocasionar danos desagradáveis ao paciente, porém, muitas vezes também são acompanhados por uma involução gradual ${ }^{4-}$ 6,19,25,29. A maior parte destas lesões não pode ser reconhecida ao nascimento, entretanto, pode surgir depois durante as oito primeiras semanas de vida.

Incidem com frequência na boca, sendo as principais áreas de ocorrência a mucosa jugal, o palato, e principalmente a língua e lábio ${ }^{26}$. Aparentemente sua etiologia ainda é incerta, sendo que estímulos endócrinos e inflamatórios podem acionar essa proliferação anormal, que em alguns casos pode ser também herdada. O hemangioma é comumente detectado ao nascimento ou na infância, mas pode ser também adquirido, decorrente de traumas ${ }^{2,3,20}$.

Clinicamente, pode apresentar-se como uma bolha, mácula ou nódulo de conteúdo sanguinolento, de coloração avermelhada ou arroxeada, relacionando sua cor com a profundidade de sua localização no tecido ${ }^{1-}$ 3,16,32. Seu tamanho pode variar de alguns milímetros a vários centímetros, apresentando-se na maioria dos casos assintomáticos ${ }^{16}$. A principal queixa relatada de pacientes portadores de hemangiomas é o distúrbio estético causado ${ }^{26}$. A diascopia ou vitropressão constitui importante auxiliar no estabelecimento do diagnóstico diferencial. À compressão feita pela lâmina de vidro, faz com que a lesão adquira coloração pálida momentaneamente, diminuindo de tamanho devido ao esvaziamento vascular e posteriormente regressando ao seu volume primitivo após a remoção da lâmina, podendo-se assim, estabelecer o diagnóstico clínico sugestivo, eliminando hipóteses de quaisquer lesões pigmentadas ${ }^{3,26,32}$. Sua consistência elástica ou fibrosa está na vinculação da quantidade de tecido conjuntivo que se interpõe entre os espaços vasculares.

De acordo com a análise histológica, podem ser classificados como capilar ou cavernoso ${ }^{26}$. O hemangioma capilar é a forma mais observada, podendo ser de origem congênita ou traumática, com tendência à involução espontânea durante os primeiros anos de vida $^{12}$. Ao exame microscópico, observa-se uma proliferação de capilares localizados superficialmente na pele ou na mucosa. A forma cavernosa é menos frequente. Apresenta-se maior em profundidade e não costuma regredir ${ }^{26}$. Caracteriza-se pela presença de vasos proliferantes de diâmetros mais amplos ${ }^{7,12}$. Apresenta grandes seios, forrados por única camada endotelial, 
podendo apresentar um componente capilar em sua superfície e um cavernoso nas porções profundas da lesão ${ }^{26,30}$. Quando localizados no assoalho da boca, podem ocasionar problemas funcionais, associados à fala, mastigação e deglutição, acarretando episódios de sangramento. Na ausência de consequências estéticas ou funcionais, a proservação é a melhor conduta a ser $\operatorname{adotada}^{26}$.

Em relação ao tratamento, deve-se ponderar sua extensão e localização, podendo intervir de maneira conservadora ou ativa, com emprego de medicamentos ou procedimentos cirúrgicos $^{3,8,17,20}$. Devido às altas taxas de involução espontânea, a proservação é a conduta mais frequentemente estabelecida, excetuando-se de situações emergenciais ${ }^{4,8,26,31}$. Atualmente, há uma tendência para o tratamento ativo de lesões que evoluem com complicações frequentes, como infecções, sangramentos e ulcerações, ou aquelas localizadas em áreas anatômicas onde a involução costuma ser mais lenta ou incompleta, como nos lábios, na ponta nasal e região de parótida, assim como em lesões mais extensas que geralmente evoluem com cicatrizes desfigurantes ${ }^{8,17}$. De acordo com a

\section{CASO CLÍNICO}

Paciente L.B.S.D, 29 anos de idade, leucoderma, gênero masculino, solicitou atendimento em consultório particular, tendo como queixa principal " mancha roxa " na literatura analisada, descreve-se que em lesões maiores e/ ou intraósseas, o tratamento deve ser realizado através da embolização ou obliteração da lesão e dos vasos adjacentes, se necessário ${ }^{1,15}$. Para as lesões menores e periféricas pode-se optar por esclerose química, excisão cirúrgica convencional, laserterapia, radioterapia, eletrocoagulação ou crioterapia $^{28}$. Independente do método de tratamento de escolha cabe ressaltar que o procedimento deve ser realizado com o mínimo de cuidados assim como qualquer outro procedimento cirúrgico ${ }^{24}$.

Analisando casos clínicos de pacientes portadores desta neoplasia vascular, há controvérsia quanto ao tratamento descrito na literatura, dai o surgimento de ambas as partes em relatar a forma de tratamento pouco exercida entre os profissionais. O objetivo deste trabalho é apresentar o relato de um caso clínico de um paciente portador de hemangioma lingual com remoção cirúrgica por enucleação mostrando uma técnica cirúrgica e rápida comparando com outras técnicas, que pode ser indicada em casos de alterações menores.

língua (FIG 1). Na oroscopia observou-se uma lesão em região lateral ventral de língua do lado esquerdo ligeiramente elevada, bem delimitada com dimensões entre 15 X $10 \mathrm{~mm}$ 
aproximadamente, de coloração arroxeada, circunscrita e séssil. Durante a anamnese foi realizado o exame de diascopia (FIG. 2) verificando-se uma diminuição da coloração vermelha. A lesão era assintomática e flácida à palpação, sem relato de outros sintomas sistêmicos. O paciente relatou que a lesão surgiu à aproximadamente sete meses e apresentava-se com crescimento progressivo. A hipótese diagnóstica sugerida foi hemangioma. $\mathrm{O}$ tratamento de escolha foi o cirúrgico, submetendo o paciente à remoção da lesão por enucleação e sutura da região (FIG 4). A peça cirúrgica removida (FIG 3) foi encaminhada para avaliação histopatológica no Centro de Patologia Oral da Faculdade de Odontologia de Piracicaba (FOP - UNICAMP), no qual foi confirmado o

Figura 1- Aspecto clínico inicial da lesão.

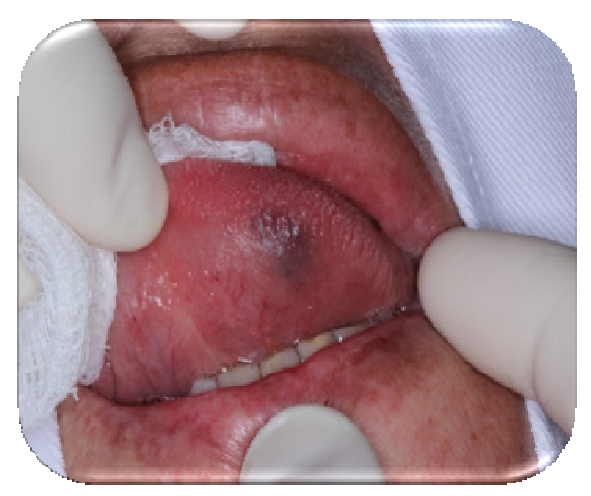

diagnóstico clínico inicial de Hemangioma Cavernoso Lingual. No pós-operatório de 30 dias, foi observada a cicatrização da região. $\mathrm{O}$ paciente encontra-se em proservação atualmente. Não foi visualizado nenhum sinal de recidiva da lesão, dentro do período de controle de seis meses. Para a descrição deste caso clínico, inicialmente foi solicitada, à paciente, autorização, através da assinatura do Termo de Consentimento Livre e Esclarecido (TCLE), e explicado os objetivos do trabalho. Em seguida, foram dadas informações garantindo o sigilo do seu nome e da sua privacidade, além de terem sido observados os princípios éticos da pesquisa que envolve seres humanos, preconizados pelo Conselho Nacional de Saúde, de acordo com a Resolução 196/96.

Figura 2 - Diascopia evidenciando a perda da coloração arroxeada momentaneamente durante a compressão.

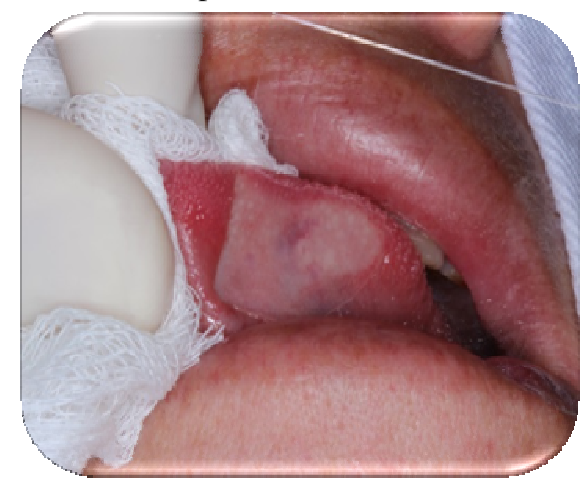


Figura 3 - Peça cirúrgica após remoção evidenciando o tecido epitelial de revestimento.

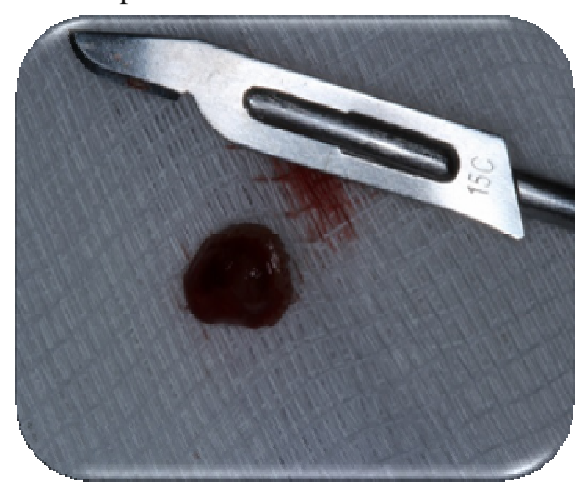

Figura 4 - Região cirúrgica após remoção da lesão com sutura de pontos isolados.

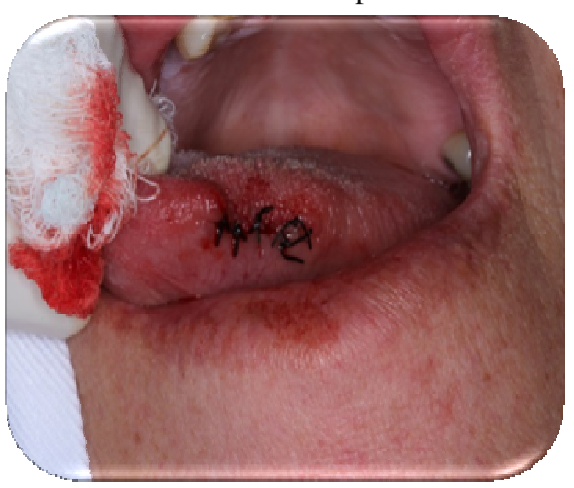

Fonte: Professor DIAS, M.A.

\section{DISCUSSÃO}

O hemangioma pode ser caracterizado como um tumor benigno proveniente da proliferação anormal de vasos sanguíneos. Rocha (2000) compartilha seu ponto de vista ao descrever o "hemangioma como sendo um hamartoma e não uma verdadeira neoplasia". Porém, tem sido relatado na literatura que, ao contrário dos hemangiomas, as malformações vasculares são descritas como anomalias estruturais de vasos sanguíneos sem proliferação endotelial, estando presentes ao nascimento e persistindo por toda vida ${ }^{18,21}$.

O caso clínico descrito apresentava características que ao exame de diascopia, possibilitou o diagnóstico clínico de hemangioma, pelo qual a positividade é sugerida pela compreensão da lâmina de vidro originando o esmaecimento da coloração inicial arroxeada, sugerindo sua origem intravascular. Sendo o exame clínico inicial de diascopia uma alternativa de diagnóstico diferencial com outras lesões como mucoceles e $\operatorname{cistos}^{5,11}$. O tratamento de escolha é relatado na literatura parecendo haver controvérsias quanto à indicação cirúrgica devido às possíveis complicações ${ }^{6,10,13,19,26,29,32}$. A localização da lesão pode induzir a procedimentos com resultados indesejáveis, como por exemplo, induzir a hemorragias, representando risco para o paciente. Existem regiões de maior risco, como a gengiva e palato dificultando a realização de quaisquer técnicas cirúrgicas ${ }^{9,21}$.

As terapias para as lesões vasculares são múltiplas e demandam ainda muitas discussões e estudos. Não há um tratamento universalmente aceito, mas sim aquele indicado para cada caso, respeitando as características da lesão e as necessidades individuais de cada paciente. Diversos métodos de tratamento vêm sendo utilizados 
na tentativa de controlar o crescimento e acelerar a regressão desta lesão pausadamente. A indicação de intervenção cirúrgica é feita através do correto diagnóstico, não devendo ser confundido com malformações vasculares, além da avaliação de localização, bem como o tamanho da lesão, além de se considerar a experiência profissional em se realizar cirurgia em lesões de alto risco hemorrágico ${ }^{21}$.

Porém, a prática cirúrgica é realizada simultaneamente à escleroterapia como opção de tratamento conservador ${ }^{22,23,27}$.Dentre as opções de tratamento, a que vem sendo mais utilizada e com grande taxa de sucesso é a escleroterapia, sem a necessidade de intervenção cirúrgica, alcançando resultados satisfatórios clinicamente e esteticamente, sendo uma opção viável e de baixo custo para os casos de hemangioma da cavidade bucal. Eliminando casos de lesões maiores e emergenciais, podendo apresentar possíveis complicações estéticas e comprometimento de funções normais como ação motora e sensitiva da região ${ }^{14,23,27,32}$.

\section{REFERÊNCIAS BIBLIOGRÁFICAS}

1. Assis, G.; Silva, S.; Moraes, P.; Amaral, J.; Germano, A. Hemangioma de língua: relato de caso. Rev Cir Traumatol BucoMaxilo-fac 2009 abr.-jun; 9(2):59.
Para Lloret (2004) $)^{14}$, a excisão cirúrgica é a técnica mais adequada relatada e na maioria das vezes o procedimento mais apropriado de eleição para o tratamento do hemangioma, principalmente quando a lesão apresenta complicação estética e compromete funções normais do paciente. A cirurgia realizada isoladamente proporciona bons resultados ${ }^{11,26}$, devendo-se evitar incisar diretamente sobre a lesão, apropriando o corte a uma margem de segurança apropriada, evitando assim a ocorrência de sangramentos abundantes e risco de recidiva da lesão ${ }^{11}$.

No presente caso, o tamanho e a localização, somados às boas condições sistêmicas do paciente, permitiram instituir procedimento cirúrgico com bisturi convencional, sem associação a qualquer agente esclerosante. $\mathrm{O}$ prognóstico se mostrou favorável, com cicatrização satisfatória da área operada e sem recorrência durante o retorno e acompanhamentos periódicos de seis meses. 
Pathol, Oral Radiol, Endod

2000; 90:731-8.

3. Boraks, S. Diagnóstico bucal. São

Paulo: Artes Médicas; 2001.

4. Brown, T.J.; Friedman, J.; Levy, M.L. The diagnosis and treatment of common birthmarks. Clin Plast Surg. 1998; 25(4):509-25.

5. Carramaschi, F.; Ferreira, M.C.; Goldenberg, D.C.; Camargo, C.P.; Faria, J.C.M; Freitas, J.M. Tratamento dos angiomas do lábio. Rev. Hosp. Clin. Fac. Med. São Paulo 46 (3): 128-132, 1991.

6. Castro, A. L. Estomatologia. $3^{\mathrm{a}}$ Ed. São Paulo: Ed. Santos, 2000. $243 p$.

7. Chinen, A.; Martins, R.H.; Santos, G.G.; Souza, A.; Marcucci, G. Hemangioma: aspectos clínicos, diagnóstico e terapêutica de 235 casos. Rev Odontol UNICID, 1996; 8:43-9.

8. Drolet, B.A.; Esterly, N.B.; Frieden, I.J. Hemangiomas in children. N Engl J Med. 1999; 341(3):173-81.
9. Eivazi, B.; Ardelean, M.; Baumler, W.; Berlien, H.P.; Cremer, H.; Elluru, R.; Koltai, P.; Olofsson, J.; Richter, G.; Schick, B.; Werner, J.A. Update on hemangiomas and vascular malformations of the head and neck. Eur Arch Otorhinolaryngol (2009) 266: 187-197.

10. Gomez, Z. Hemangiomas tratamiento: resultado de 15 casos. Acta Odontol Venez, v.22, n.2, p.105-120, may/ aug. 1984.

11. Hernández, C.C. Hemangiomas revision bibliográfica de esta afeccion. Rev. Cub. Estomatol. 23 (3): 235-243, 1986.

12. Leão, J.C.; Gones, D.O. Alterações vasculares. Disponibilidade: www.ufpe.br/estomatologia/ lesoes_pigmentadas.htm-44K. [03 maio. 2014].

13. Ling, K.C. Slerotherapy of oral haemangioma with $3 \%$ sodium tetradecil sulphate: a case report. Singapore Med J, v.27, n.3, p. 244-246, jun. 1986. 
14. Lloret, P. Tratamiento médico de los hemangiomas. An Sist Sanit Navar. 2004; 27 (Suppl 1): 81-92.

15. Loureiro, C.C.; Falchet, P.C.; Gavranich, J.Jr.; Lobo Leandro, L.F. Embolization as the treatment for a life-threatening mandibular arteriovenous malformation. $\mathbf{J}$ Craniofac Surg. 2010 Mar; 21(2):380-2.

16. Marcucci, G. Estomatologia. Rio de Janeiro: Editora Guanabara Koogan, 2005. p. 139-140.

17. Metry, D.W.; Hebert, A.A. Benign cutaneous vascular tumors of infancy: when to worry, what to do. Arch Dermatol. 2000; 136(7):905-14.

18. Neville, B.; Damm, D.; Allen, C.; Bouquot, J. Patologia oral \& maxilofacial. Rio de Janeiro:

Guanabara Koogan; 1998.

19. Neville, B.W.; Damm, D.D.; Allen, C.M.; Bouquot, J.E. Patologia Oral e Maxilofacial: $2^{\text {a. }}$.Ed. Rio de Janeiro: Guanabara Koogan, 2004.
20. Pagnocelli, R.M.; Sant'Ana Filho, M. Tratamento do Hemangioma capilar. Rev Fac Odontol Porto Alegre, 1994; 35 (1): 2-5.

21. Palacios, C.J.; Herrera, C.P.; Lugo, M.V. La escleroterapia como una alternativa en el tratamiento de los hemangiomas de los tejidos blandos de La cavidad bucal. Acta Odontol Venez, 2000; 38 (2): 4-8.

22. Pedron, I.G.; Carnaval, T.G.; Loureiro, C.C.S.; Utumi, E.R.; Magalhães, J.C.A.; Adde, C.A. Opção terapêutica de hemangioma labial. Rev Inst Ciênc Saúde. 2008; 26 (4): 477-81.

23. Regezi, S. A.; Sciubba, J. J. Lesões vermelho azuis. In: Patologia Bucal: correlações clinicopatológicas. Rio de Janeiro: Guanabara Koogan, 1991. p. 86-90.

24. Rocha, L.B.; Pádua, J.M.; Martins, R.H.; Lia, R.C.C. Hemangioma da cavidade bucal. RGO 2000; 48:150:2. 
25. Sadeghi, E.; Gingrass, D. Oral hemangioma treated with a sclerosing agent. Int $\mathbf{J}$ Oral Maxillofac Surg, 1989; 18 (5): 262-3.

26. Seo, J., Utumi, E., Zambon, C., Pedron, I., Rocha, A. Escleroterapia de hemangioma labial. Rev Odonto 2009 juldez;17(34):106.

27. Shafer, W. G., Hine, M. K., Levy, B. M. Tratado de Patologia Bucal. Rio de Janeiro: Guanabara Koogan, 1987. p. 143-144.

28. Spetic, M.B.S.A. Avaliação da presença de mastócitos em hemangioma e granulomas piogênicos bucais. Dissertação de Mestrado, F.O.B. USP, 2001.
Cellular markers that distinguish the phases of hemangioma during infancy and childhood. J Clin Invest. 1994;93(6):2357-64.

30. Toledo, H, Castro, E, Castro, A, Soubhia, A, FB SJ. Hemangioma cavernoso de lábio inferior: caso clínico. Revista Odontológica de Araçatuba 2004 jan-jun; 25(1):9.

31. Tommasi, A.F. (Ed). Diagnóstico em patologia bucal. 2a . Ed. São Paulo: Pancast, 1989. Pag.247248.

32. Wang, L., Oliveira, D.T., Consolaro, A., Perez, F. Tratamento de hemangioma bucal com agente esclerosante. Tratamento de hemangioma bucal com agente esclerosante. ROBRAC 1998;7:20-2.

29. Takahashi, K., Mulliken, J.B., Kozakewich, H.P., Rogers, R.A., Folkman, J., Ezekowitz, A.B. 